\title{
ON THE CLASSIFICATION PROBLEM FOR RANK 2 TORSION-FREE ABELIAN GROUPS
}

\author{
ALEXANDER S. KECHRIS
}

\section{Introduction}

We study here some foundational aspects of the classification problem for torsionfree abelian groups of finite rank. These are, up to isomorphism, the subgroups of the additive groups $\left(\mathbb{Q}^{n},+\right)$, for some $n=1,2,3, \ldots$ The torsion-free abelian groups of rank $\leqslant n$ are the subgroups of $\left(\mathbb{Q}^{n},+\right)$.

For $n=1$, that is, the subgroups of $(\mathbb{Q},+)$, the isomorphism problem was solved by Baer in the 1930s (see [10]). For every torsion-free abelian group $G, x \in G, x \neq 0$, and $p \in P=$ the set of primes, let

and put

$$
h_{p}(x)=\sup \left\{n \in \mathbb{N}: p^{n} \text { divides } x\right\} \in\{0,1,2, \ldots, \infty\},
$$

For $h, g \in\{0,1, \ldots, \infty\}^{P}$, let

$$
h(x)=\left(h_{p}(x)\right)_{p \in P} .
$$

$$
\begin{array}{r}
h \sim g \Leftrightarrow h, g \text { agree on the } \infty \text { coordinates and } \\
h(p) \neq g(p) \text { for only finitely many } p .
\end{array}
$$

It is not hard to see that if $G$ has rank 1, then for $x, y \neq 0$ in $G, h(x) \sim h(y)$. We then define the type of $G, \tau(G)$, by

$$
\tau(G)=\text { the } \sim \text {-equivalence class of any } h(x), x \in G, x \neq 0 .
$$

Then $\tau(G)$ is a complete invariant of isomorphism of rank 1 torsion-free abelian groups.

THEOREM 1 (Baer). If $G, G^{\prime}$ are torsion-free abelian groups of rank 1, then

$$
G \cong G^{\prime} \Leftrightarrow \tau(G)=\tau\left(G^{\prime}\right) .
$$

For torsion-free abelian groups of rank 2 or higher, however, no satisfactory classification has been found and this has been one of the main problems in this area (see [10], Section 93, Problem 66, p. 183]). Recently, in [15], a different approach has been proposed, which aims to show that no reasonably simple classification is possible by precisely quantifying the difficulty of this classification problem and characterizing the set-theoretic complexity of any potential complete invariants.

In general, a classification problem consists of a family of objects $X$ and an equivalence relation $E$ on $X$. A complete classification of $X$ up to $E$ consists of finding a set of invariants $I$ and a map $c: X \longrightarrow I$ such that $x E y \Leftrightarrow c(x)=c(y)$. For this to be of any interest both $I$ and $c$ must be as explicit and concrete as possible.

Received 15 June 1999; revised 16 November 1999.

2000 Mathematics Subject Classification 20K15.

Research partially supported by NSF Grant DMS 9619880.

J. London Math. Soc. (2) 62 (2000) 437-450 (c) London Mathematical Society 2000. 
Quite often in mathematical practice the class of objects $X$ can be viewed as forming a standard Borel space (that is, a Polish space equipped with its Borel structure) and $E$ turns out to be a Borel equivalence relation on $X$. The theory of Borel equivalence relations studies the set-theoretic nature of possible complete invariants for Borel equivalence relations and develops a mathematical framework for measuring the complexity of such classification problems (see [20] for a general discussion of these issues).

The following notion is basic in organizing this study. Let $E, F$ be Borel equivalence relations on standard Borel spaces $X, Y$ respectively. We say that $R$ is Borel reducible to $F$, in symbols

$$
E \leqslant{ }_{\mathrm{B}} F,
$$

if there is a Borel map $f: X \longrightarrow Y$ such that

$$
x E y \Leftrightarrow f(x) F f(y) .
$$

Intuitively, this means that the classification problem for $E$ is, in some sense, at most as complicated as that of $F$, since any complete invariants for $F$ work as well for $E$ (after composing with $f$ ). Also let

$$
E \sim_{\mathrm{B}} F \Leftrightarrow E \leqslant_{\mathrm{B}} F \text { and } F \leqslant_{\mathrm{B}} E .
$$

Intuitively, this means that $E$ and $F$ have classification problems of equal complexity. Finally put

$$
E<{ }_{\mathrm{B}} F \Leftrightarrow E \leqslant_{\mathrm{B}} F \text { and } F \Varangle_{\mathrm{B}} E \text {, }
$$

which means that $E$ has a (strictly) simpler classification problem than $F$.

Another way to understand the meaning of $E \leqslant_{\mathrm{B}} F$ is to notice that it simply expresses that there is an embedding of $X / E$ into $Y / F$ which is 'Borel', in the sense that it has a Borel lifting (from $X$ into $Y$ ). Thus $E \leqslant_{\mathrm{B}} F$ means that $X / E$ has 'Borel cardinality' less than or equal to that of $Y / F$, so one can view this as the study of the Borel cardinality of the quotient spaces $X / E$. Thus $E \sim_{\mathrm{B}} F$ means that $X / E, Y / F$ have the same Borel cardinality and $E<_{\mathrm{B}} F$ means that $X / E$ has (strictly) smaller Borel cardinality than $Y / F$.

Going back now to torsion-free abelian groups, let $S\left(\mathbb{Q}^{n}\right)$ be the standard Borel space of all subgroups of $\left(\mathbb{Q}^{n},+\right)$, which is a closed subset of the compact, metric space $\mathscr{P}\left(\mathbb{Q}^{n}\right)\left(\equiv 2^{\mathbb{Q}^{n}}\right)$ of all subsets of $\mathbb{Q}^{n}$. If $\mathrm{GL}_{n}(\mathbb{Q})$ denotes the countable group of all $n \times n$ invertible matrices with entries in $\mathbb{Q}$, then $\mathrm{GL}_{n}(\mathbb{Q})$ acts by linear transformations on $\mathbb{Q}^{n}$ and thus on $S\left(\mathbb{Q}^{n}\right)$, and it is easy to see that if we denote by

$$
\cong_{n}
$$

the isomorphism relation on torsion-free abelian groups of rank $\leqslant n$, then, for $G, G^{\prime} \in S\left(\mathbb{Q}^{n}\right)$,

$$
G \cong{ }_{n} G^{\prime} \Leftrightarrow \exists M \in \mathrm{GL}_{n}(\mathbb{Q})\left(M \cdot G=G^{\prime}\right),
$$

that is, $\cong_{n}$ is induced by this action of $\mathrm{GL}_{n}(\mathbb{Q})$ on $S\left(\mathbb{Q}^{n}\right)$. Since this action is clearly Borel, it follows that $\cong_{n}$ is a Borel equivalence relation, and since $\mathrm{GL}_{n}(\mathbb{Q})$ is a countable group, it follows that $\cong_{n}$ is a countable equivalence relation, that is, every equivalence class is countable.

We now use the theory of countable Borel equivalence relations to formulate some precise problems and results concerning the complexity of $\cong_{n}$. First, we will briefly review the general structure of the (pre)order $\leqslant_{B}$ on countable Borel equivalence relations (for which we refer to $[\mathbf{2 0}]$ for a general survey, and $[\mathbf{8}, \mathbf{1 6}]$ for detailed developments). 
The simplest countable Borel equivalence relations are the so-called concretely classifiable ones (also called smooth or tame), where $E$ on $X$ is concretely classifiable if there is a Borel map $f: X \longrightarrow Y, Y$ a standard Borel space, such that $x E y \Leftrightarrow f(x)=$ $f(y)$. This means that one can find complete invariants for $E$ which are members of a standard Borel space, and thus fairly concrete. Denoting by $X$ also the equality relation on a standard Borel space $X$ and by $n$ any standard Borel space of finite cardinality $n$, the concretely classifiable $E$ are, up to $\sim_{\mathrm{B}}$, simply those in the list

$$
1<_{\mathrm{B}} 2<_{\mathrm{B}} 3 \ldots<_{\mathrm{B}} n<_{\mathrm{B}} \ldots<_{\mathrm{B}} \mathbb{N}<_{\mathrm{B}} \mathbb{R},
$$

and any non-concretely classifiable countable Borel equivalence relation $E$ satisfies

$$
\mathbb{R}<{ }_{\mathrm{B}} E .
$$

Among those there is a smallest one, in the sense of $\leqslant_{\mathrm{B}}$, namely $E_{0}$ defined on $2^{\mathbb{N}}$ by

$$
x E_{0} y \Leftrightarrow \exists n \forall m \geqslant n\left(x_{m}=y_{m}\right) .
$$

This is a special case of the so-called Glimm-Effros dichotomy (see $[\mathbf{9}, \mathbf{1 2}, \mathbf{1 3}]$ ). Finally, among all the countable Borel equivalence relations there is a largest one, in the sense of $\leqslant_{\mathrm{B}}$, denoted by $E_{\infty}$ and called universal. One realization of $E_{\infty}$ is given by the equivalence relation induced by the translation action of $F_{2}$, the free group with two generators, on its subsets. Another is the isomorphism relation on locally finite trees. (A tree is a connected acyclic graph and it is locally finite if every vertex has only finitely many neighbors.) It turns out that

$$
E_{0}<{ }_{\mathrm{B}} E_{\infty} .
$$

Thus all non-concretely classifiable countable Borel equivalence relations $E$ fall in the interval

between $E_{0}$ and $E_{\infty}$.

$$
E_{0} \leqslant{ }_{\mathrm{B}} E \leqslant{ }_{\mathrm{B}} E_{\infty},
$$

There are also intermediate countable Borel equivalence relations $E$,

$$
E_{0}<{ }_{\mathrm{B}} E<{ }_{\mathrm{B}} E_{\infty},
$$

the most studied ones being the so-called treeable ones (originally introduced in [2] in the context of ergodic theory). A countable Borel equivalence relation $E$ on $X$ is called treeable if there is a Borel acyclic graph $R$ on $X$ whose connected components are the $E$-equivalence classes. It turns out that there is, in the sense of $\leqslant_{B}$, a largest treeable countable Borel equivalence relation, denoted by

$$
E_{\infty \mathrm{T}} \text {. }
$$

One realization of $E_{\infty \mathrm{T}}$ is the isomorphism relation on rigid (that is, having only the identity as automorphism) locally finite trees. It turns out that

$$
E_{0}<{ }_{\mathrm{B}} E_{\infty \mathrm{T}}<{ }_{\mathrm{B}} E_{\infty},
$$

and that the treeable non-tame countable Borel equivalence relations are those in the interval

$$
E_{0} \leqslant{ }_{\mathrm{B}} E \leqslant{ }_{\mathrm{B}} E_{\infty \mathrm{T}} .
$$

We can now try to measure the complexity of $\cong_{n}$ by placing it in this hierarchy of countable Borel equivalence relations under $\leqslant_{\mathrm{B}}$.

First we note that Baer's theorem easily implies the following.

Proposition 2 (folklore). $\left(\cong_{1}\right) \sim_{\mathrm{B}} E_{0}$. 
Thus $\cong_{1}$ is at the bottom of this hierarchy of non-concretely classifiable countable Borel equivalence relations.

By contrast, in [15] it was conjectured that for $n \geqslant 2$ the isomorphism relation $\cong_{n}$ is at the other extreme of this hierarchy, that is, universal.

CONJECTURE 3 (Hjorth and Kechris [15]). For $n \geqslant 2,\left(\cong_{n}\right) \sim_{\mathrm{B}} E_{\infty}$.

A proof of this conjecture would quantify the exact set-theoretic complexity of any possible complete invariants for rank 2 or higher torsion-free abelian groups and would show that any complete classification in this case would have to be quite complicated. (Such classifications do exist, but are considered unsatisfactory precisely because of their complexity, certainly in comparison with the rank 1 case; see [10].)

The first step in that direction was taken by Hjorth [14], who showed the following.

THEOREM 4 (Hjorth [14]). (i) $E_{0}<_{\mathrm{B}}\left(\cong_{2}\right)$.

(ii) For $n \geqslant 3, \cong_{n}$ is not treeable.

Hence, in a precise sense, the classification problem for rank 2 or higher torsionfree abelian groups is more complicated than that of rank 1 groups, and it is even more so for rank 3 or higher torsion-free abelian groups.

This result raises the natural question of whether $\cong_{2}$ is treeable, and the main result here is that this fails as well, so the same phenomenon occurs for all torsionfree abelian groups of rank 2 .

THEOREM $5 . \cong_{2}$ is not treeable.

REMARK 6. The proof in [14] actually shows that the isomorphism relation on the torsion-free abelian groups of rank exactly $n, n \geqslant 3$, is not treeable, and our proof here also shows the same thing for $n=2$.

The proof of Theorem 5 is based on a general result on the non-treeability of the equivalence relations induced by certain actions of a class of countable groups, which is interesting in its own right. Hjorth's [14] proof of Theorem 4(ii) is also based on a result of this type, due to Adams and Spatzier [5]. In order to state succinctly these results it will be convenient to introduce the following terminology, as in [11].

Definition 7. Let $G$ be a countable group. We call $G$ antitreeable if for every Borel action of $G$ on a standard Borel space $X$, which is free and admits an invariant probability Borel measure, the corresponding equivalence relation $E_{G}^{X}$ is not treeable.

The first examples of antitreeable groups were discovered by Adams and Spatzier [5], who showed that any Kazhdan (or property $(T)$ ) group $G$ is antitreeable. Typical examples of Kazhdan groups are the groups $\mathrm{SL}_{n}(\mathbb{Z})$, and $\operatorname{PSL}_{n}(\mathbb{Z})=$ the quotient of $\mathrm{SL}_{n}(\mathbb{Z})$ by its center $\left(=\mathrm{SL}_{n}(\mathbb{Z})\right.$, if $n$ is odd, $=\left(\mathrm{SL}_{n}(\mathbb{Z}) /\left\{I_{n},-I_{n}\right\}\right.$, where $I_{n}=$ the identity $n \times n$ matrix), if $n$ is even), for $n \geqslant 3$; see [7].

Using this notion, let us summarize Hjorth's argument for Theorem 4(ii).

We have $\mathrm{SL}_{n}(\mathbb{Z}) \subseteq \mathrm{GL}_{n}(\mathbb{Q})$, and since $\mathrm{SL}_{n}(\mathbb{Z})$ preserves $\mathbb{Z}^{n}$, it acts on

$$
S\left(\mathbb{Q}^{n}, \mathbb{Z}^{n}\right)=\left\{G \in S\left(\mathbb{Q}^{n}\right): G \supseteq \mathbb{Z}^{n}\right\} .
$$


Since the center of $\mathrm{SL}_{n}(\mathbb{Z})$ leaves every element of $S\left(\mathbb{Q}^{n}, \mathbb{Z}^{n}\right)$ fixed, the group $\operatorname{PSL}_{n}(\mathbb{Z})$ acts also on $S\left(\mathbb{Q}^{n}, \mathbb{Z}^{n}\right)$. Using duality theory for the discrete group $\mathbb{Q}^{n} / \mathbb{Z}^{n}$, Hjorth defines then a probability Borel measure $v_{n}$ on $S\left(\mathbb{Q}^{n}, \mathbb{Z}^{n}\right)$ with the following properties:

(i) $v_{n}$ is $\operatorname{PSL}_{n}$ (Z)-invariant.

(ii) The action is free on a Borel invariant set of $v_{n}$-measure 1 .

Hence by the antitreeability of $\operatorname{PSL}_{n}(\mathbb{Z})$ for $n \geqslant 3$, it follows that the isomorphism relation on $S\left(\mathbb{Q}^{n}, \mathbb{Z}^{n}\right)$ is not treeable, and thus so is $\cong_{n}$ for $n \geqslant 3$, since treeability of an equivalence relation is preserved by going to its restriction to a Borel set and also by going to a subequivalence relation (see $[\mathbf{1 1}, \mathbf{1 6}]$ ).

REMARK 8. Since all groups in $S\left(\mathbb{Q}^{n}, \mathbb{Z}^{n}\right)$ have rank exactly $n$, it also follows that $\cong_{n}$ restricted to groups of rank exactly $n$ is not treeable, if $n \geqslant 3$.

Unfortunately, this proof does not work for $n=2$. Not only is the group $\operatorname{PSL}_{2}(\mathbb{Z})$ not antitreeable but actually the opposite is true: every free action of $\mathrm{PSL}_{2}(\mathbb{Z})$ gives rise to a treeable equivalence relation (see [16]).

What turns out to work is to replace $\operatorname{PSL}_{2}(\mathbb{Z})$ by $\operatorname{PSL}_{2}(\mathbb{Z}[1 / 2])$, where $\mathbb{Z}[1 / 2]$ is the ring of dyadic rationals. In other words consider now the action of $\operatorname{PSL}_{2}(\mathbb{Z}[1 / 2])$ on the set $S\left(\mathbb{Q}^{2}, \mathbb{Z}[1 / 2]^{2}\right)$ of all subgroups of $\mathbb{Q}^{2}$ containing $\mathbb{Z}[1 / 2]^{2}$. Then the argument of Hjorth [14] works and is unchanged for this action and it produces a probability Borel measure $v_{2}^{\prime}$ on $S\left(\mathbb{Q}^{2}, \mathbb{Z}[1 / 2]^{2}\right)$ which is invariant under the action of $\operatorname{PSL}_{2}(\mathbb{Z}[1 / 2])$ and such that this action is free $v_{2}^{\prime}$-almost-everywhere. Thus, as before, Theorem 5 will follow if we can show that $\operatorname{PSL}_{n}(\mathbb{Z}[1 / 2])$ is antitreeable for all $n \geqslant 2$.

Recall that if $G$ is a locally compact second countable group and $\Gamma \subseteq G$ is a subgroup, we call $\Gamma$ a lattice in $G$ if $\Gamma$ is discrete and there is an invariant Borel probability measure for the canonical action of $G$ on $G / \Gamma: g \cdot h \Gamma=g h \Gamma$. It is now a standard result that $\Gamma=\operatorname{PSL}_{n}(\mathbb{Z}[1 / p]), p$ a prime number, is a lattice in the product group $G=\operatorname{PSL}_{n}(\mathbb{R}) \times \operatorname{PSL}_{n}\left(\mathbb{Q}_{p}\right)$, where $\mathbb{Q}_{p}$ is the $p$-adic field. (Here $g \in \Gamma$ is identified with $(g, g) \in G$.) In fact, more generally, a result of Borel asserts that if $G$ is a connected semisimple $\mathbb{Q}$-group, then $G(\mathbb{Z}[1 / p])$ is a lattice in $G(\mathbb{R}) \times G\left(\mathbb{Q}_{p}\right)$ (see $[\mathbf{2 3}$, 10.1.1]). Notice also that $\operatorname{PSL}_{n}(\mathbb{Z}[1 / 2]) \supseteq \operatorname{PSL}_{n}(\mathbb{Z})$ is not an amenable group, if $n \geqslant 2$, and $\operatorname{PSL}_{n}(\mathbb{R}), \operatorname{PSL}_{n}\left(\mathbb{Q}_{p}\right)$ each contain an infinite amenable discrete subgroup, for example

$$
\left\{\left(\begin{array}{cc}
p^{n} & 0 \\
0 & p^{-n}
\end{array}\right): n \in \mathbb{Z}\right\} .
$$

Thus the result that $\operatorname{PSL}_{n}(\mathbb{Z}[1 / 2])$ is antitreeable for $n \geqslant 2$ is an immediate corollary of the following theorem.

THEOREM 9. Let $\Gamma$ be a countable group which is a lattice in a product $G=G_{1} \times G_{2}$ of two locally compact second countable groups, each of which contains an infinite amenable discrete subgroup. Then, if $\Gamma$ is not amenable, $\Gamma$ is antitreeable.

The proof of this result (or rather the stronger statement given in Theorem 10 below) is based on the method used in [1]. Gaboriau [11] has also proved this result in the case when $\Gamma=\Gamma_{1} \times \Gamma_{2}$ is a product of two infinite countable groups, using the method of 'costs'. 
Actually we derive Theorem 9 from an even more general result, which might also be of some interest. Recall that a countable Borel equivalence relation $E$ is hyperfinite if and only if $E \leqslant{ }_{\mathrm{B}} E_{0}$.

THEOREM 10. Let $G_{1}, G_{2}$ be locally compact second countable groups, each containing an infinite discrete amenable subgroup. Let $G=G_{1} \times G_{2}$ and consider a Borel action of $G$ on a standard Borel space $Y$, which is free and admits an invariant Borel probability measure $\lambda$. If $E$ is a treeable countable Borel equivalence relation on a standard Borel space $X$ and $\pi: Y \longrightarrow Y$ is a Borel function reducing $E_{G}^{Y}$ to $E$ (that is, $\left.x E_{G}^{Y} y \Leftrightarrow \pi(x) E \pi(y)\right)$, then

(i) if $\pi_{*}(\lambda)=\mu, E$ is hyperfinite on an invariant Borel set of $\mu$-measure 1;

(ii) $G$ is amenable.

REMARK 11. In connection with the hypotheses of the preceding theorems, we should point out that there are infinite countable groups $\Gamma$ which contain no infinite amenable subgroups. The following example was pointed out to us by Gaboriau. Olshanski [21] has proved that every non-cyclic torsion-free hyperbolic group $H$ has an infinite homomorphic image $\Gamma$ all of whose proper subgroups are finite. Thus if one takes such an $H$ that is Kazhdan, $\Gamma$ is not amenable (because the image of a homomorphism from a Kazhdan group in an amenable group is finite) and so has no infinite amenable subgroups.

We conclude this introduction with some comments concerning rigid groups. We call an abelian group rigid if its only automorphisms are $x \longmapsto \pm x$. We denote by $\cong_{n}^{*}$ the isomorphism relation on rigid groups of rank $\leqslant n$. Strengthening Hjorth's result, Thomas [22] showed that $E_{0}<_{\mathrm{B}}\left(\cong_{2}^{*}\right)$, and for $n \geqslant 3$, ${ }_{n}^{*}$ is not treeable. (It is not hard to see that $\left(\cong_{1}^{*}\right) \sim_{\mathrm{B}} E_{0}$.) In [3] it was shown that for all $n \geqslant 1,\left(\cong_{n}^{*}\right)<_{\mathrm{B}}\left(\cong_{n+1}^{*}\right)$, so, in particular, $\left(\cong{ }_{n}^{*}\right)<_{\mathrm{B}} E_{\infty}$ for all $n$, that is, none of the $\cong_{n}^{*}$ is universal. It is of course still open whether $\cong_{n}$, for $n \geqslant 2$, is universal. In an earlier draft of this paper, we erroneously claimed that $\simeq_{2}^{*}$ is also not treeable, but A. Hales pointed out an error in this argument. Inspection of the proof in [14] shows that the measure $v_{2}^{\prime}$ concentrates on groups whose automorphisms are exactly the maps $x \longmapsto \pm 2^{n} x$ for an integer $n$, so that they have automorphism group $\mathbb{Z}_{2} \times \mathbb{Z}$. This shows that $\cong_{2}$ restricted to groups with this automorphism group is not treeable. It leaves open however the problem of whether $\cong_{2}^{*}$ is treeable.

\section{Proofs I}

First we derive Theorem 9 from Theorem 10. This uses the standard method of induced actions.

Suppose $\Gamma$ is a lattice in a locally compact second countable group $G$. Let $\Gamma$ act in a Borel way on a standard Borel space $X$ with invariant probability Borel measure $\mu$. Then we can define the induced action of $G$ on a space $Y$ (to be defined presently) as follows.

Let $T$ be a Borel transversal for $G / \Gamma$ containing 1 (see $[19,12.17])$. This is a Borel set $T \subseteq G$ which meets every left coset $g \Gamma$ in exactly one point and contains 1 . We identify $T$ with $G / \Gamma$ via $t \longrightarrow t \Gamma$. Then the canonical action of $G$ on $G / \Gamma$ is transferred to the following action of $G$ on $T$ :

$$
g \cdot t=\text { the unique element of } T \text { in } g t \Gamma .
$$


By assumption, there is an invariant Borel probability measure on $T$ for this action, call it $v$. Let also

$$
\begin{aligned}
\rho(g, t) & =(\text { the unique element } \gamma \in \Gamma \text { such that }(g \cdot t) \gamma=g t) \\
& =(g \cdot t)^{-1} g t .
\end{aligned}
$$

Then $\rho: G \times T \longrightarrow \Gamma$ is a Borel cocycle, that is, it satisfies

$$
\rho(h g, t)=\rho(h, g \cdot t) \rho(g, t) \text {. }
$$

We now take $Y=X \times T$ and define the induced action of $G$ on $Y$ by

$$
g \cdot(x, t)=(\rho(g, t) \cdot x, g \cdot t) .
$$

Let also $\lambda=\mu \times v$. Then the following are easy to verify:

(i) $\lambda$ is $G$-invariant (this follows from Fubini).

(ii) If the $\Gamma$-action on $X$ is free, the $G$-action on $Y$ is free.

(iii) $\pi(x, t)=x$ is a Borel reduction of $E_{G}^{Y}$ to $E_{\Gamma}^{X}$, that is, $(x, t) E_{G}^{Y}(y, s) \Leftrightarrow x E_{\Gamma}^{X} y$.

(iv) $\pi_{*}(\lambda)=\mu$.

Thus if $\Gamma$ is a non-amenable lattice in a product $G=G_{1} \times G_{2}$ as in Theorem 10 and $\Gamma$ is not antitreeable, one can find a Borel action of $\Gamma$ on some standard Borel space $X$ such that the action is free, has an invariant Borel probability measure $\mu$, and $E_{\Gamma}^{X}$ is treeable. Then consider the induced action of $G$ on $Y$, and the associated $\lambda, \pi$. Then all the hypotheses of Theorem 10 are valid, so, by (ii) of that theorem, $G$ must be amenable, and thus $\Gamma$ must be amenable, a contradiction.

REMARK 12. For the particular $G, Y, \pi, \lambda$ we use here, we could have also obtained a contradiction by using instead (i) of Theorem 10 , because $E=E_{\Gamma}^{X}$ is hyperfinite on a set of $\mu$-measure 1 , which implies that $\Gamma$ is amenable, since the $\Gamma$-action is free and has the invariant probability measure $\mu$ (see, for example, $[17,2.3($ ii) $])$.

\section{Proofs II}

We prove here Theorem 10(i). Throughout the rest of this section we agree on the convention that measure means probability Borel measure.

First we claim that we can assume that $E$ is treeable by trees of degree $\leqslant 3$, that is, that there is an acyclic Borel graph such that each vertex has at most three neighbors, so that the $E$-classes are its connected components. To see this, we claim that $E \leqslant{ }_{\mathrm{B}} E^{\prime}$, where $E^{\prime}$ (on $X^{\prime}$ ) is treeable by trees of degree $\leqslant 3$. Granting this and fixing a Borel function $p: X \longrightarrow X^{\prime}$ reducing $E$ to $E^{\prime}$, we note that $p \circ \pi$ reduces $E_{G}^{Y}$ to $E^{\prime}$, so if the result holds for $E^{\prime}$, then $E^{\prime}$ is hyperfinite on a Borel set of $(p \circ \pi)_{*}(\lambda)$ measure 1 , so $E$ is hyperfinite on a $\pi_{*}(\lambda)$-measure 1 Borel set.

The claim, which comes from [16, §3], can be proved as follows. Fix an acyclic Borel graph $R$ on $X$ whose connected components are the $E$-classes. Let $I(\mathbb{N})=\mathbb{N} \times \mathbb{N}$ be the largest equivalence relation on $\mathbb{N}$. Then clearly $E \leqslant_{\mathrm{B}} E^{\prime}=E \times I(\mathbb{N})$. To see that $E^{\prime}$ has the property that we need, fix a countable group $H=\left(h_{n}\right)_{n \in \mathbb{N}}$ of Borel automorphisms on $X$ inducing $E$. On $X^{\prime}=X \times \mathbb{N}$ define a graph as follows: $(x, m)$ is connected by an edge to $(y, n)$ if either $x=y$ and $|m-n|=1$ or else $x, y$ are connected by an edge in the graph $R$ and $e(x, y)=m, e(y, x)=n$, where $e(x, y)=$ least $m\left(h_{m} \cdot x=y\right)$. This clearly works. 
Denote by $T_{C}, C \in X / E$, the tree associated to the equivalence class $C$ of $E$. Thus the vertex set of $T_{C}$ is $C$. Also $T_{C}$ has degree $\leqslant 3$. Since $B=\left\{x \in X:[x]_{E}\right.$ is finite $\}$ is a Borel set and $E \mid B$ is tame (and thus hyperfinite) it follows that we can also assume that $E$ is aperiodic, that is, every $E$-class is infinite. Thus if we denote by $\partial T_{C}$ the (compact, metric) space of ends of $T_{C}$ (see [19]) then $\partial T_{C} \neq \varnothing$, for all $C \in X / E$.

It will also be convenient, although hardly necessary, to assume the continuum hypothesis below. This does not cause any harm since the statement we are trying to prove is a projective statement, so, by a general metamathematical result, if it can be proved, for example, using the continuum hypothesis it can be also proved without the continuum hypothesis (for a convenient reference see, for example, [4]).

Below we identify $G_{1}$ with $G_{1} \times\{1\} \subseteq G$ and $G_{2}$ with $\{1\} \times G_{2} \subseteq G$, so that we view $G_{1}, G_{2}$ as subgroups of $G$.

Now fix infinite discrete amenable subgroups $\Gamma_{i} \subseteq G_{i}$ and put $E_{i}=E_{\Gamma_{i}}^{Y}\left(\subseteq E_{G}^{Y}\right)$ for $i=1,2$. Also, by Mokobodzki's theorem (see, for example, [17, proof of Fact 2.3, (i)], using the continuum hypothesis, fix a universally measurable finitely additive probability measure, $\Phi_{i}$, on $\Gamma_{i}$, which is $\Gamma_{i}$-invariant.

We will often consider below maps $M(y)$ with arguments $y \in Y$ and values $M(y)$ which are either measures on $\partial T_{[\pi(y)]_{E}}$ or finite subsets of $\partial T_{[\pi(y)]_{E}}$. Given a class $\mathscr{F}$ of functions between standard Borel spaces, we want to define what it means to say that such an $M$ is in the class $\mathscr{F}$. Consider for example the case when $M(y)$ is a measure on $\partial T_{[\pi(y)]_{E}}$ (the other case being similar).

For each $x \in X$ fix an injection $f_{x}:[x]_{E} \longrightarrow 3^{<N}$ which is an isomorphism between the rooted tree $T_{[x]_{E}}$ with root $x$ and a subtree $U_{x}$ of $3^{<N}$ (with root $\varnothing$ ). This is done in a canonical way so that the map $f(x, y)=f_{x}(y)$ from $E$ into $3^{\mathbb{N}}$ is Borel. This induces a homeomorphism $g_{x}$ from $\partial T_{[x]_{E}}$ onto $\left[U_{x}\right]$ ( = the set of all branches of $U_{x}$ ) which is a closed subspace of $3^{\mathbb{N}}$. For each compact, metric space $K$ denote by $P(K)$ the compact, metrizable space of measures on $K$ with the weak*-topology. We now say that a function $M$ with domain $Y$ such that $M(y) \in P\left(\partial T_{[\pi(y)]_{E}}\right)$ is in a class $\mathscr{F}$, if the function $\hat{M}: Y \longrightarrow P\left(3^{\mathbb{N}}\right)$ given by $\hat{M}(y)=g_{\pi(y)}(M(y))$, where $g_{\pi(y)}(M(y))$ is the image of $M(y)$ by the homeomorphism $g_{\pi(y)}$ of $\partial T_{[\pi(y)]_{E}}$ onto $\left[U_{\pi(y)}\right] \subseteq 3^{\mathbb{N}}$, is in the class $\mathscr{F}$.

We will break up the rest of the proof into a sequence of steps.

Step I: For $i=1,2$, there is a map $S_{i}^{0}$ which assigns to each $y \in Y$ a non-empty finite subset $S_{i}^{0}(y) \subseteq \partial T_{[\pi(y)]_{E}}$ such that

(i) $S_{i}^{0}$ is universally measurable;

(ii) $\left|S_{i}^{0}(y)\right| \leqslant 2$;

(iii) $S_{i}^{0}$ is $\Gamma_{i}$-invariant.

To see this fix a Borel function $b(y)$ which assigns to each $y \in Y$ some $b(y) \in$ $\partial T_{[\pi(y)]_{E}}$. Then define a universally measurable function $M_{i}(y)$ which assigns to each $y \in Y$ a measure $M_{i}(y)$ on $\partial T_{[\pi(y)]_{E}}$ as follows.

For $p$ a continuous real function on $\partial T_{[\pi(y)]_{E}}$, consider the map

$$
\gamma \in \Gamma_{i} \longmapsto p(b(\gamma \cdot y))
$$

(note that $[\pi(y)]_{E}=[\pi(\gamma \cdot y)]_{E}$ ), and let

$$
\Lambda_{y}^{i}(p)=\int p(b(\gamma \cdot y)) d \Phi_{i}(\gamma)
$$

Then $\Lambda_{y}^{i}$ is a positive linear functional on $C\left(\partial T_{[\pi(y)]_{E}}\right)$, with $\Lambda_{y}^{i}(1)=1$, so we can 
identify it with a measure $M_{i}(y)$ on $\partial T_{[\pi(y)]_{E}}$. The $\Gamma_{i}$-invariance of $\Phi_{i}$ implies that $M_{i}(y)=M_{i}(\gamma \cdot y)$ for all $\gamma \in \Gamma_{i}$. The universal measurability of $\Phi_{i}$ implies that of $M_{i}$.

For each triple $\left(e_{1}, e_{2}, e_{3}\right)$ of distinct points of $\partial T_{C}$, denote by

$$
\psi\left(e_{1}, e_{2}, e_{3}\right)
$$

the unique point in $C$ which is at the intersection of the lines

$$
\left[e_{1}, e_{2}\right],\left[e_{2}, e_{3}\right],\left[e_{3}, e_{1}\right] \text {. }
$$

The argument below comes from [4]. Using Fubini, we can easily see that for every measure $v$ on a standard Borel space $W$, either $v^{3}\left(\left\{(x, y, z) \in W^{3}: x, y, z\right.\right.$ are distinct $\})>0$ or else $v$ is supported by at most two points of $W$, that is, for a subset $S$ of $W$ of cardinality at most 2 , we have $v(S)=1$. We apply this to each $M_{i}(y)$.

If $M_{i}(y)^{3}$ gives positive measure to the set of distinct triples $\left(e_{1}, e_{2}, e_{3}\right)$ from $\partial T_{[\pi(y)]_{E}}$, then, using $\psi$ as above, we can map $M_{i}(y)^{3}$ restricted to this set (after normalizing) to a measure $\rho_{y}^{i}$ on $[\pi(y)]_{E}$, which is a countable set. This allows us to pick, using $\rho_{y}^{i}$, a finite subset of $[\pi(y)]_{E}$, namely the set of points of maximum measure in $\rho_{y}^{i}$, and thus a single element $s_{i}(y)$ of $[\pi(y)]_{E}$, and so also a single element $x_{i}(y) \in \partial T_{[\pi(y)]_{E}}$. We then put in this case $S_{i}^{0}(y)=\left\{x_{i}(y)\right\}$.

Otherwise, $M_{i}(y)$ is supported by at most two points, so we let $S_{i}^{0}(y)=$ $\operatorname{support}\left(M_{i}(y)\right)$.

It is easy to check that all this can be done so that (i)-(iii) of Step I are satisfied.

Step II: Suppose that $y \longmapsto \mu_{y}$ is such that $\mu_{y} \in P\left(\partial T_{[\pi(y)]_{E}}\right)$, and $y \longmapsto \mu_{y}$ is $\lambda$ measurable, and $\Gamma_{i}$-invariant $\lambda$-almost-everywhere (that is, for all $\gamma \in \Gamma_{i}\left(\mu_{\gamma \cdot y}=\mu_{y}\right)$, $\lambda$-almost-everywhere $(y))$. Then $\mu_{y}$ is supported by at most two points $\lambda$-almosteverywhere.

Indeed, otherwise there is a Borel set $A \subseteq\left\{y: \mu_{y}\right.$ is not supported by at most two points $\}$ such that $A$ is $\Gamma_{i}$-invariant and $\lambda(A)>0$. By the argument used in Step I, there is a $\lambda$-measurable function $g: A \longrightarrow X$ such that $g$ is $\Gamma_{i}$-invariant and $g(y) \in[\pi(y)]_{E}$. By shrinking $A$, if necessary, we can assume that $g$ is actually Borel.

We now claim that $E_{i} \mid A$ is tame, that is, it admits a Borel selector, which is absurd as $E_{i} \mid A$ is induced by a free action of $\Gamma_{i}$ with invariant measure. To prove this claim we argue as follows. If $E_{i} \mid A$ is not tame, then by the Glimm-Effros dichotomy, it admits a non-atomic $E_{i}$-ergodic measure, say $m$. Then there is a Borel set $B \subseteq A$ which is $\Gamma_{i}$-invariant, has $m$-measure 1 , and $g \mid B$ is constant, say with value $x_{0}$. Then $\pi(B) \subseteq\left[x_{0}\right]_{E}$, so $B \subset G \cdot y_{0}$, for some $y_{0} \in Y$. Thus, in particular, $E_{i} \mid\left(G \cdot y_{0}\right)$ is not tame. On the other hand, there is a natural bijection between the quotient space of $E_{i} \mid\left(G \cdot y_{0}\right)$ and the space of right cosets $\Gamma_{i} \backslash G$ of $\Gamma_{i}$ in $G$, namely $\Gamma_{i} g \longmapsto \Gamma_{i} \cdot\left(g \cdot y_{0}\right)$. Since $\Gamma_{i}$ is closed in $G, \Gamma_{i} \backslash G$ is a standard Borel space, so this shows that $E_{i} \mid\left(G \cdot y_{0}\right)$ is tame, a contradiction.

Step III: Now consider the set

of $\lambda$-measurable functions $S$ which satisfy the following conditions:

(i) $S(y)$ is a non-empty, finite subset of $\partial T_{[\pi(y)]_{E}}$, $\lambda$-almost-everywhere $(y)$.

(ii) $|S(y)| \leqslant 2, \lambda$-almost-everywhere $(y)$.

(iii) $S$ is $\lambda$-measurable and $\Gamma_{i}$-invariant, $\lambda$-almost-everywhere (that is, for all $\gamma \in$ $\Gamma_{i}(S(\gamma \cdot y)=S(y)), \lambda$-almost-everywhere $\left.(y)\right)$. 
Since $S_{i}^{0} \in \mathscr{S}_{i}, \mathscr{S}_{i} \neq \varnothing$. Next define the partial order $\leqslant$ on $\mathscr{S}_{i}$ by $S \leqslant T \Leftrightarrow S(y) \subseteq T(y), \quad \lambda$-almost-everywhere $(y)$.

Claim 13. $\left(\mathscr{S}_{i}, \leqslant\right)$ has a maximum element.

Proof. For $S \in \mathscr{S}_{i}$, let

$$
D(S)=\{y:|S(y)|=2\} .
$$

Put $r=\sup \left\{\lambda(D(S)): S \in \mathscr{S}_{i}\right\}$. We first argue that this sup is attained. To see this, pick $S_{n} \in \mathscr{S}_{i}$ with $\lambda\left(D\left(S_{n}\right)\right)>r-(1 / n)$. Let

$$
\mu_{n}(y)= \begin{cases}\frac{1}{2}\left(\delta_{e_{1}(y)}+\delta_{e_{2}(y)}\right), & \text { if } S_{n}(y)=\left\{e_{1}(y), e_{2}(y)\right\}, \\ \delta_{e(y)}, & \text { if } S_{n}(y)=\{e(y)\}\end{cases}
$$

where $\delta_{e}=$ the Dirac measure at $e$. Thus $\mu_{n}$ is defined $\lambda$-almost-everywhere, and is $\Gamma_{i^{-}}$ invariant $\lambda$-almost-everywhere. Let

$$
\mu_{\infty}(y)=\sum 2^{-n} \mu_{n}(y)
$$

so that $\mu_{\infty}$ has the same properties. Then by Step II, $\mu_{\infty}(y)$ is supported by at most two points, $\lambda$-almost-everywhere $(y)$, say $S_{\infty}(y)$. Thus $S_{\infty} \in \mathscr{S}_{i}$. Also $D\left(S_{n}\right) \subseteq D\left(S_{\infty}\right)$; thus $\lambda\left(D\left(S_{\infty}\right)\right)=r$.

We show that this $S_{\infty}$ works, that is, $S \leqslant S_{\infty}$, for all $S \in \mathscr{S}_{i}$. Indeed, fix such a $S$. By a similar argument to the above, $S \cup S_{\infty} \in \mathscr{S}_{i}$, so $D(S) \subseteq D\left(S_{\infty}\right) \lambda$-almosteverywhere, as $D(S) \subseteq D\left(S \cup S_{\infty}\right)=D\left(S_{\infty}\right) \lambda$-almost-everywhere. Also

and

$$
\lambda\left(\left\{y:|S(y)|=1 \text { and }\left|S_{\infty}(y)\right|=2 \text { and } S(y) \nsubseteq S_{\infty}(y)\right\}\right)=0 \text {, }
$$

$$
\lambda\left(\left\{y:|S(y)|=1 \text { and }\left|S_{\infty}(y)\right|=\text { and } S(y) \nsubseteq S_{\infty}(y)\right\}\right)=0,
$$

since otherwise we would have $\lambda\left(D\left(S \cup S_{\infty}\right)\right)>r$. Hence $S \subseteq S_{\infty} \lambda$-almost-everywhere.

Clearly this maximum element is unique $\lambda$-almost-everywhere and we call it $S_{i}^{\infty}$.

Step IV: For $g_{2} \in G_{2}$, define

Then for $\gamma_{1} \in \Gamma_{1}$,

$$
S_{g_{2}}(y)=S_{1}^{\infty}\left(g_{2} \cdot y\right)
$$

$$
\begin{aligned}
S_{g_{2}}\left(\gamma_{1} \cdot y\right) & =S_{1}^{\infty}\left(g_{2} \cdot\left(\gamma_{1} \cdot y\right)\right) \\
& =S_{1}^{\infty}\left(\gamma_{1} \cdot\left(g_{2} \cdot y\right)\right) \\
& =S_{1}^{\infty}\left(g_{2} \cdot y\right) \\
& =S_{g_{2}}(y), \quad \lambda \text {-almost-everywhere }(y) .
\end{aligned}
$$

Thus $S_{g_{2}} \in \mathscr{S}_{1}$ and $S_{g_{2}} \subseteq S_{1}^{\infty} \lambda$-almost-everywhere. However

$$
\begin{aligned}
y \in D\left(S_{g_{2}}\right) & \Leftrightarrow\left|S_{g_{2}}(y)\right|=2 \\
& \Leftrightarrow\left|S_{1}^{\infty}\left(g_{2} \cdot y\right)\right|=2 \\
& \Leftrightarrow g_{2} \cdot y \in D\left(S_{1}^{\infty}\right),
\end{aligned}
$$

so $\lambda\left(D\left(S_{g_{2}}\right)\right)=\lambda\left(D\left(S_{1}^{\infty}\right)\right)$; thus, as before, $S_{g_{2}}=S_{1}^{\infty}, \lambda$-almost-everywhere. Hence

for all $g_{2} \in G_{2}\left[S_{1}^{\infty}(y)=S_{1}^{\infty}\left(g_{2} \cdot y\right), \lambda\right.$-almost-everywhere $\left.(y)\right]$,

and thus, in particular,

for all $\gamma_{2} \in \Gamma_{2}\left(S_{1}^{\infty}(y)=S_{1}^{\infty}\left(\gamma_{2} \cdot y\right)\right), \quad \lambda$-almost-everywhere $(y)$, 
that is, $S_{1}^{\infty}$ is also $\Gamma_{2}$-invariant $\lambda$-almost-everywhere, so $S_{1}^{\infty} \in \mathscr{T}_{2}$. Hence $S_{1}^{\infty} \subseteq S_{2}^{\infty}$ $\lambda$-almost-everywhere, and thus, by symmetry,

$$
S_{1}^{\infty}=S_{2}^{\infty}, \quad \lambda \text {-almost-everywhere. }
$$

Moreover, the above argument showed that $S_{1}^{\infty}$ is $G_{2}$-invariant $\lambda$-almost-everywhere (that is, it satisfies (1) above) and similarly $S_{2}^{\infty}$ is $G_{1}$-invariant $\lambda$-almost-everywhere, so $S_{1}^{\infty}$ is also $G_{1}$-invariant $\lambda$-almost-everywhere, and thus, since $G=G_{1} G_{2}, S_{1}^{\infty}$ is $G$ invariant $\lambda$-almost-everywhere.

Thus we have found a function $S\left(=S_{1}^{\infty}\right)$ with the following properties:

(i) $S$ is $\lambda$-measurable.

(ii) $S(y)$ is a non-empty finite subset of $\partial T_{[\pi(y)]_{E}}$ and $|S(y)| \leqslant 2$, $\lambda$-almosteverywhere $(y)$.

(iii) $S$ is $G$-invariant $\lambda$-almost-everywhere, that is, for any $g \in G$,

$$
S(g \cdot y)=S(y), \quad \lambda \text {-almost-everywhere }(y) .
$$

Step V: Let $B_{0}$ be a Borel subset of $Y$ such that $\lambda(Y)=1, S \mid B_{0}$ is Borel and $0<|S(y)| \leqslant 2$ for $y \in B_{0}$.

Below ' $\forall{ }_{\lambda}^{*} y \ldots$ ' means that ' $\lambda(\{y: \ldots\})=1$ ' and ' $\forall * g \ldots$ ' means that 'the set of $g \in G$ with ... is a Haar conull set'. Thus we have

so, by Fubini,

$$
\forall g \forall_{\lambda}^{*} y\left(g \cdot y \in B_{0}\right),
$$

Also

$$
\forall_{\lambda}^{*} y \forall^{*} g\left(g \cdot y \in B_{0}\right) \text {. }
$$

$$
\forall g \forall * \frac{\forall_{\lambda}^{*}}{y}(S(g \cdot y)=S(y))
$$

so, by Fubini again, the set

$$
\begin{aligned}
A_{0} & =\left\{y: \forall^{*} g\left(g \cdot y \in B_{0}\right) \text { and } g \longmapsto S(g \cdot y) \text { is constant on a Haar conull set }\right\} \\
& =\left\{y: \forall^{*} g\left(g \cdot y \in B_{0}\right) \text { and } \forall^{*} g \forall^{*} h(S(g \cdot y)=S(h \cdot y))\right\}
\end{aligned}
$$

in a $G$-invariant Borel set of $\lambda$-measure 1 .

For $y \in A_{0}$, let

$$
S_{0}(y)=\text { the common value of } S(g \cdot y) \text { on a Haar conull set of } g \text {. }
$$

Then $S_{0}$ is Borel and assigns to each $y \in A_{0}$ a non-empty subset $S_{0}(y)$ of $\partial T_{[\pi(y)]_{E}}$ of cardinality $\leqslant 2$ and clearly $S_{0}$ is $G$-invariant. Put

$$
C_{0}=\left[\pi\left(A_{0}\right)\right]_{E},
$$

so that $C_{0}$ is Borel and has $\pi_{*}(\lambda)=\mu$-measure 1. Fix also $p: C_{0} \longrightarrow A_{0}$, which is Borel and satisfies $\pi(p(x)) E x$. Then $T_{0}=S_{0} \circ p$ is Borel and assigns to each $x \in C_{0}$ a nonempty subset $T_{0}(x) \subseteq \partial T_{[x]_{E}}$ of cardinality $\leqslant 2$ and clearly $T_{0}$ is $E$-invariant. It follows by [16, Section 3] (see also [1]) that $E \mid C_{0}$ is hyperfinite and this concludes the proof of Theorem 10(i).

\section{Proofs III}

We finally prove Theorem 10(ii). This follows from [23, 4.3.3], since the $G$-space $Y$ turns out to be amenable, using [6]. For the convenience of the reader who is not familiar with this subject we will write out a more or less self-contained proof. 
We use the following characterization of amenability of locally compact second countable groups; see [23, 4.1.1].

$G$ is amenable if and only if for any continuous action of $G$ on a compact, metrizable space $K$, there is a $G$-invariant measure on $K$.

Fix such an action of $G\left(=G_{1} \times G_{2}\right)$ on $K$. To find an invariant measure, we claim that it is enough to find a $\lambda$-measurable function $\psi: Y \longrightarrow P(K)$ such that for every $g \in G$

$$
\psi(g \cdot y)=g \cdot \psi(y), \quad \lambda \text {-almost-everywhere }(y) .
$$

Indeed, if such a $\psi$ exists, then if $\Lambda=\psi_{*}(\lambda), \Lambda$ is a measure on $P(K)$, and if $b(\Lambda) \in P(K)$ is the barycenter of $\Lambda$, we claim that $g \cdot b(\Lambda)=b(\Lambda)$, that is, $b(\Lambda)$ is the required fixed point.

To see this, write for $\mu \in P(K), f \in C(K)$,

$$
\mu(f)=\int f d \mu .
$$

Then

$$
(g \cdot \mu)(f)=\mu(k \longmapsto f(g \cdot k)) .
$$

The measure $b(\Lambda)$ is defined by the property

so

$$
b(\Lambda)(f)=\int \mu(f) d \Lambda(\mu),
$$

$$
\begin{aligned}
b(\Lambda)(f) & =\int \mu(f) d \psi_{*}(\lambda)(\mu) \\
& =\int \psi(y)(f) d \lambda(y),
\end{aligned}
$$

and thus

$$
\begin{aligned}
(g \cdot b(\Lambda))(f) & =b(\Lambda)(k \longmapsto f(g \cdot k)) \\
& =\int \psi(y)(k \longmapsto f(g \cdot k)) d \lambda(y) \\
& =\int g \cdot \psi(y)(f) d \lambda(y) \\
& =\int \psi(g \cdot y)(f) d \lambda(y) \\
& =\int \psi(y)(f) d \lambda(y) \\
& =b(\Lambda)(f),
\end{aligned}
$$

that is, $g \cdot b(\Lambda)=b(\Lambda)$.

Hence it is enough to find such a $\psi$. Let $S$ be a countable Borel section for $E_{G}^{Y}$, that is, $S$ is a Borel set which meets every $G$-orbit in a non-empty countable set (see $[18,1.1])$. Put $R=E_{G}^{Y} \mid S$. Now $\pi$ clearly shows that $R \leqslant_{\mathrm{B}} E$, so $R$ is hyperfinite when restricted to the intersection of $S$ with some $G$-invariant Borel set of $\lambda$-measure 1 . Thus we may as well assume that $R$ is hyperfinite. Also, by the Lusin-Novikov theorem (see $[19,18.10]$ ), fix a Borel function $s: Y \longrightarrow S$ such that $s(y) E_{G}^{Y} y$ and $s(y)=y$, if $y \in S$. Let then $h(y) \in G$ be defined by

$$
h(y) \cdot y=s(y) .
$$


Finally, for $y, z$ in the same $G$-orbit, let $g(y, z) \in G$ be defined by

$$
g(y, z) \cdot y=z \text {. }
$$

Now consider the measure

$$
\kappa=s_{*}(\lambda)
$$

on $S$. Fix a countable group $\left\{g_{n}\right\}$ of Borel automorphisms of $S$ which generate $R$, that is, $x R y \Leftrightarrow$ there exists $n\left(g_{n} \cdot x=y\right)$, and let

$$
\hat{\kappa}=\sum_{n=0}^{\infty} \frac{1}{2^{n+1}}\left(g_{n} \cdot \kappa\right) .
$$

Then $\hat{\kappa}$ is a measure on $S, \hat{\kappa}(A)=\kappa(A)$, if $A$ is $R$-invariant, and $\hat{\kappa}$ is $R$-quasi-invariant (that is, the $R$-saturation of any Borel subset of $S$ of $\hat{\kappa}$-measure 0 also has $\hat{\kappa}$-measure 0 ). Since $R$ is hyperfinite, it is $\hat{\kappa}$-amenable in the sense of Zimmer, which means the following.

For any Borel cocycle $\alpha: R \longrightarrow \operatorname{LI}(B)$, into the group of linear isometries of a separable Banach space $B$, let $\alpha^{*}$ be the adjoint cocycle

$$
\alpha^{*}(s, t)=\left(\alpha(s, t)^{-1}\right)^{*} \mid H\left(B_{1}^{*}\right),
$$

where $B_{1}^{*}$ is the unit ball of $B^{*}$ and $H\left(B_{1}^{*}\right)$ its homeomorphism group, when $B_{1}^{*}$ is equipped with the weak*-topology. A Borel field is a Borel map $s \longmapsto K_{s}$ from $S$ into the compact, convex, non-empty subsets of $B_{1}^{*}$. A Borel map $\sigma: S \longrightarrow B_{1}^{*}$ is a section of $\left\{K_{s}\right\}$ if $\sigma(s) \in K_{s}, \hat{\kappa}$-almost-everywhere $(s)$. The Borel field $\left\{K_{s}\right\}$ is called $R$-invariant if for all $t\left(s R t \Rightarrow \alpha^{*}(s, t)\left(K_{s}\right)=K_{t}\right), \hat{\kappa}$-almost-everywhere $(s)$, and $\sigma$ is called $R$ invariant if for all $t\left(s R t \Rightarrow \alpha^{*}(s, t)(\sigma(s))=\sigma(t)\right), \hat{\kappa}$-almost-everywhere $(s)$. Now $\hat{\kappa}$ amenability of $R$ means that for every such $\alpha,\left\{K_{s}\right\}$ there is a Borel $R$-invariant section.

In our particular case, we apply this as follows.

Let $B=C(K)$, so that $G$ acts on $C(K)$ by linear isometries, via

$$
(g \cdot f)(k)=f\left(g^{-1} \cdot k\right) \text {. }
$$

Denote by $\bar{g}$ the linear isometry corresponding to $g \in G$. Put

$$
\begin{aligned}
\alpha(s, t) & =\overline{g(s, t)}, \\
K_{s} & =P(K) \quad\left(\text { a compact, convex, non-empty subset of } B_{1}^{*}\right) .
\end{aligned}
$$

Then find a Borel $R$-invariant $\sigma: S \longrightarrow B_{1}^{*}$ such that $\sigma(s) \in P(K), \hat{\kappa}$-almost-everywhere $(s)$. Since $\hat{\kappa}$ is $R$-quasi-invariant, we can find an $R$-invariant Borel set $S_{0} \subseteq S$ of $\hat{\kappa}$ measure 1 such that $\sigma(s) \in P(K)$, and also $\alpha^{*}(s, t)(\sigma(s))=\sigma(t)$ for every $s, t \in S_{0}$ with $s R t$. This means that $g(s, t) \cdot \sigma(s)=\sigma(t)$ for $s, t \in S_{0}$ with $s R t$. Now $\hat{\kappa}\left(S_{0}\right)=1$ implies that $\kappa\left(S_{0}\right)=1$, since $S_{0}$ is $R$-invariant; thus, if $Y_{0}=s^{-1}\left(S_{0}\right)$, then $\lambda\left(Y_{0}\right)=1$ and $Y_{0}$ is $G$-invariant.

Now we claim that if, for $y \in Y_{0}$, we put

this works. Indeed,

$$
\psi(y)=h(y)^{-1} \cdot \sigma(s(y)),
$$

$$
\psi(g \cdot y)=h(g \cdot y)^{-1} \cdot \sigma(s(g \cdot y))
$$

so we only have to check that

or

$$
g \cdot h(y)^{-1} \cdot \sigma(s(y))=h(g \cdot y)^{-1} \cdot \sigma(s(g \cdot y))
$$

which is clear, as

$$
h(g \cdot y) \cdot g \cdot h(y)^{-1} \cdot \sigma(s(y))=\sigma(s(g \cdot y)),
$$

$$
g(s(y), s(g \cdot y))=h(g \cdot y) g h(y)^{-1} .
$$


Acknowledgements. I would like to thank S. Adams and G. Hjorth for many helpful conversations and $\mathrm{G}$. Mess for bringing up the idea of using groups of the form $\operatorname{PSL}_{n}\left(\mathbb{Z}\left[1 / p_{1}, \ldots, 1 / p_{n}\right]\right)$ instead of $\operatorname{PSL}_{n}(\mathbb{Z})$.

\section{References}

1. S. AdAms, 'Indecomposability of treed equivalence relations', Israel J. Math. 64 (1988) 362-380.

2. S. Adams, 'Trees and amenable equivalence relations', Ergodic Theory Dynam. Systems 10 (1990) $1-14$.

3. S. AdAms and A. S. Kechris, 'Linear algebraic groups and countable Borel equivalence relations', J. Amer. Math. Soc. (2000) (to appear).

4. S. Adams and R. Lyons, 'Amenability, Kazhdan's property and percolation for trees, groups, and equivalence relations', Israel J. Math. 75 (1991) 341-370.

5. S. Adams and R. Spatzier, 'Kazhdan groups, cocycles and trees', Amer. J. Math. 112 (1990) 271-287.

6. S. Adams, G. A. Elliott and T. Giordano, 'Amenable actions of groups', Trans. Amer. Math. Soc. 344 (1994) 803-822.

7. P. De la Harpe and A. Valette, 'La propriété $(T)$ de Kazhdan pour les groupes localement compacts', Astérisque 175 (1989).

8. R. Dougherty, S. JACKSON and A. S. KeChris, 'The structure of hyperfinite Borel equivalence relations', Trans. Amer. Math. Soc. 341 (1994) 193-225.

9. E. G. EFfros, 'Transformation groups and C*-algebras', Ann. of Math. 81 (1965) 38-55.

10. L. Fuchs, Infinite Abelian groups (Academic Press, 1970).

11. D. Gaboriau, 'Coût des relations d'équivalence et des groupes', Invent. Math. 139 (2000) 41-98.

12. J. Glimm, 'Locally compact transformation groups', Trans. Amer. Math. Soc. 101 (1961) 124-138.

13. L. Harrington, A. S. Kechris and A. Louveau, 'A Glimm-Effros dichotomy for Borel equivalence relations', J. Amer. Math. Soc. 3 (1990) 903-928.

14. G. HJORTH, 'Around nonclassifiability for countable torsion-free abelian groups', preprint, 1998

15. G. Huorth and A. S. Kechris, 'Borel equivalence relations and classifications of countable models', Ann. Pure Appl. Logic 82 (1996) 221-272.

16. S. JACKSON, A. S. KeChris and A. Louveau, 'Countable Borel equivalence relations', preprint, 2000.

17. A. S. Kechris, 'Amenable equivalence relations and Turing degrees', J. Symbolic Logic 56 (1991) $182-194$.

18. A. S. Kechris, 'Countable sections for locally compact group actions, Ergodic Theory Dynam. Systems 12 (1992) 283-295.

19. A. S. Kechris, Classical descriptive set theory, Graduate Texts in Mathematics 156 (Springer, 1995).

20. A. S. Kechris, 'New directions in descriptive set theory', Bull. Symbolic Logic 5 (1999) 161-179.

21. G. OlshANSKI, 'On residualing homomorphisms and $G$-subgroups of hyperbolic groups', Internat. $J$. Algebra Comput. 3 (1993) 365-409.

22. S. Thomas, 'Notes on TFA, groups', preprint, 1998.

23. R. Zimmer, Ergodic theory and semisimple groups (Birkhäuser, 1984).

Department of Mathematics

Caltech

Pasadena

CA 91125

USA 